\title{
HOW A NONASSOCIATIVE ALGEBRA REFLECTS THE PROPERTIES OF A SKEW POLYNOMIAL
}

\author{
C. BROWN AND S. PUMPLÜN
}

\begin{abstract}
Let $D$ be a unital associative division ring and $D[t ; \sigma, \delta]$ be a skew polynomial ring, where $\sigma$ is an endomorphism of $D$ and $\delta$ a left $\sigma$-derivation. For each $f \in D[t ; \sigma, \delta]$ of degree $m>1$ with a unit as leading coefficient, there exists a unital nonassociative algebra whose behaviour reflects the properties of $f$. These algebras yield canonical examples of right division algebras when $f$ is irreducible. The structure of their right nucleus depends on the choice of $f$. In the classical literature, this nucleus appears as the eigenspace of $f$, and is used to investigate the irreducible factors of $f$. We give necessary and sufficient criteria for skew polynomials of low degree to be irreducible. These yield examples of new division algebras $S_{f}$.
\end{abstract}

\section{INTRODUCTION}

The investigation of skew polynomials is an active area in algebra which has applications to coding theory, to solving differential and difference equations, and in engineering, to name just a few. For instance, linear differential operators (where $\sigma=i d$ ) and linear difference operators (where $\delta=0$ ) are special cases of skew polynomials.

Let $D$ be a unital associative division ring and $R=D[t ; \sigma, \delta]$ a skew polynomial ring, where $\sigma$ is an endomorphism of $D$ and $\delta$ a left $\sigma$-derivation. Suppose $f \in D[t ; \sigma, \delta]$ has degree $m$. Using right division by $f$ to define a multiplication on the set of skew polynomials of degree less than $m$, this set becomes a unital nonassociative algebra we denote by $S_{f}$. The algebra $S_{f}$ generalizes the classical quotient algebra construction when factoring out a twosided ideal generated by a right invariant skew polynomial $f$. When choosing $f$ and $R$ in the right way, it can be also seen as a generalization of certain crossed product algebras and some Azumaya algebra constructions. First results on the structure of the algebras $S_{f}$ which initially were defined by Petit in [30] have appeared in [30, 31, 5, 6, 35, 36, 34]. First applications to coding theory have appeared for instance in [37, 38, 39].

Recently, a computational criterion for deciding whether a bounded skew polynomial is irreducible was developed in [17]. The method heavily relies on being able to find the zero divisors in the right nucleus of $S_{f}$ (although the simple algebra employed there, called the eigenspace of $f$, is not recognized as the right nucleus of $S_{f}$ in that paper). The method is only applicable for certain set-ups when the input data $S, \sigma$ and $\delta$ are effective and computable, but it demonstrates the importance of developing a better understanding of the algebras $S_{f}$ and their algebraic structure. Independently, effective algorithms to compute

2010 Mathematics Subject Classification. Primary: 16S36; Secondary: 17A60, 17A99.

Key words and phrases. Skew polynomials, skew polynomial ring, Ore polynomials, nonassociative algebra. 
the eigenspace (and thus the right nucleus of $S_{f}$, again not recognized as such) for the special case that $R=\mathbb{F}_{q}(x)[t ; \sigma, \delta]$ can be found in [14], and for $R=\mathbb{F}_{q}[t ; \sigma]$ in [13], [40]. In all cases the eigenspace is a crucial tool to understand the decomposability of the skew polynomial $f$.

This paper consists of two parts. The first one considers the structure of the right nucleus of the algebras $S_{f}$, establishing how it reflects the type of the skew polynomial $f$ it is defined with, but also the important role irreducible polynomials play in the construction of classes of nonassociative unital (right) division algebras.

The second part looks at skew polynomials of low degree as well as the polynomial $f(t)=$ $t^{m}-a$, and when these polynomials are irreducible in $D[t ; \sigma, \delta]$, in order to obtain examples for the construction of (right) division algebras.

After establishing the basic terminology in Section 1, we define Petit algebras in Section 2 and collect some results on their right nuclei in Section 3. We investigate when the algebras $S_{f}$ are right (and not left) division algebras in Section 4. A necessary condition for $S_{f}$ being a right division algebra is that the polynomial $f$ is irreducible. We then collect some irreducibility criteria for polynomials of low degree and the polynomial $f(t)=t^{m}-a$ in both $R=D[t ; \sigma]$ and $R=D[t ; \sigma, \delta]$ in Sections 5 and 6 , including the special case where $D$ is a finite field.

We point out that there exists some kind of Eisenstein valuation criteria to test a skew polynomial over a division ring for reducibility, using some (noncommutative) valuation theory for skew polynomial rings $[11,18]$. We believe our criteria are more tractable for the types of skew polynomials we consider. Moreover, some results on twisted polynomials over algebraically and real closed fields have been obtained in [2] and [32, 33].

Most of this work is part of the first author's PhD thesis [4] written under the supervision of the second author.

\section{Preliminaries}

1.1. Skew polynomial rings. Let $S$ be a unital associative ring, $\sigma$ a ring endomorphism of $S$ and $\delta: S \rightarrow S$ a left $\sigma$-derivation, i.e. an additive map such that $\delta(a b)=\sigma(a) \delta(b)+\delta(a) b$ for all $a, b \in S$. Then the skew polynomial ring $R=S[t ; \sigma, \delta]$ is the set of skew polynomials $g(t)=a_{0}+a_{1} t+\cdots+a_{n} t^{n}$ with $a_{i} \in S$, with term-wise addition and where the multiplication is defined via $t a=\sigma(a) t+\delta(a)$ for all $a \in S[29]$. That means,

$$
a t^{n} b t^{m}=\sum_{j=0}^{n} a\left(\Delta_{n, j} b\right) t^{m+j}
$$

for all $a, b \in S$, where the map $\Delta_{n, j}$ is defined recursively via

$$
\Delta_{n, j}=\delta\left(\Delta_{n-1, j}\right)+\sigma\left(\Delta_{n-1, j-1}\right),
$$

with $\Delta_{0,0}=i d_{S}, \Delta_{1,0}=\delta, \Delta_{1,1}=\sigma$. Therefore $\Delta_{n, j}$ is the sum of all monomials in $\sigma$ and $\delta$ of degree $j$ in $\sigma$ and degree $n-j$ in $\delta$ [20, p. 2]. If $\delta=0$, then $\Delta_{n, n}=\sigma^{n}$.

For $\sigma=i d$ and $\delta=0$, we obtain the usual ring of left polynomials $S[t]=S[t ; i d, 0]$. Define $\operatorname{Fix}(\sigma)=\{a \in S \mid \sigma(a)=a\}$ and $\operatorname{Const}(\delta)=\{a \in S \mid \delta(a)=0\}$. 
For $f(t)=a_{0}+a_{1} t+\cdots+a_{n} t^{n} \in R$ with $a_{n} \neq 0$ define $\operatorname{deg}(f)=n$ and $\operatorname{deg}(0)=-\infty$. Then $\operatorname{deg}(g h) \leq \operatorname{deg}(g)+\operatorname{deg}(h)$ (with equality if $h$ has an invertible leading coefficient, or $g$ has an invertible leading coefficient and $\sigma$ is injective, or if $S$ is a division ring). An element $f \in R$ is irreducible in $R$ if it is not a unit and it has no proper factors, i.e if there do not exist $g, h \in R$ with $1 \leq \operatorname{deg}(g), \operatorname{deg}(h)<\operatorname{deg}(f)$ such that $f=g h$.

1.2. Nonassociative algebras. Let $R$ be a unital commutative ring and let $A$ be an $R$ module. We call $A$ an algebra over $R$ if there exists an $R$-bilinear map $A \times A \mapsto A$, $(x, y) \mapsto x \cdot y$, usually denoted simply by juxtaposition $x y$, the multiplication of $A$. An algebra $A$ is called unital if there is an element in $A$, denoted by 1 , such that $1 x=x 1=x$ for all $x \in A$. We will only consider unital algebras.

For an $R$-algebra $A$, associativity in $A$ is measured by the associator $[x, y, z]=(x y) z-$ $x(y z)$. The left nucleus of $A$ is defined as $\operatorname{Nuc}_{l}(A)=\{x \in A \mid[x, A, A]=0\}$, the middle nucleus as $\operatorname{Nuc}_{m}(A)=\{x \in A \mid[A, x, A]=0\}$ and the right nucleus as $\operatorname{Nuc}_{r}(A)=\{x \in$ $A \mid[A, A, x]=0\} . \operatorname{Nuc}_{l}(A), \operatorname{Nuc}_{m}(A)$ and $\operatorname{Nuc}_{r}(A)$ are associative subalgebras of $A$. Their intersection $\operatorname{Nuc}(A)=\{x \in A \mid[x, A, A]=[A, x, A]=[A, A, x]=0\}$ is the nucleus of $A$. $\operatorname{Nuc}(A)$ is an associative subalgebra of $A$ containing $R 1$ and $x(y z)=(x y) z$ whenever one of the elements $x, y, z$ is in $\operatorname{Nuc}(A)$. The commuter of $A$ is defined as $\operatorname{Comm}(A)=\{x \in$ $A \mid x y=y x$ for all $y \in A\}$ and the center of $A$ is $\mathrm{C}(A)=\operatorname{Nuc}(A) \cap \operatorname{Comm}(A)$ [41].

A nonassociative ring $A \neq 0$ (resp., an algebra $A \neq 0$ over a field $F$ ) is called a left division ring (resp., algebra), if for all $a \in A, a \neq 0$, the left multiplication with $a, L_{a}(x)=a x$, is a bijective map, and a right division ring (resp., algebra), if for all $a \in A, a \neq 0$, the right multiplication with $a, R_{a}(x)=x a$, is a bijective map. An algebra $A \neq 0$ over a field $F$ is called a division algebra if for all $a \in A, a \neq 0$, both the left and right multiplication with $a$ are bijective. A division algebra $A$ does not have zero divisors. If $A$ is a finite-dimensional algebra over $F$, then $A$ is a division algebra over $F$ if and only if $A$ has no zero divisors [41]. A nonassociative ring $A \neq 0$ has no zero divisors if and only if $R_{a}$ and $L_{a}$ are injective for all $0 \neq a \in A$.

Note that every algebra $A$ is a right $\operatorname{Nuc}_{r}(A)$-module and the left multiplication $L_{a}$ is $\operatorname{Nuc}_{r}(A)$-linear for all $0 \neq a \in A$.

\section{Nonassociative algebras obtained from SKew polynomials}

Let $S$ be a unital associative ring and $S[t ; \sigma, \delta]$ a skew polynomial ring where $\sigma$ is injective.

2.1. Assume $f(t)=\sum_{i=0}^{m} a_{i} t^{i} \in R=S[t ; \sigma, \delta]$ has an invertible leading coefficient $a_{m} \in S^{\times}$. Then for all $g(t) \in R$ of degree $l \geq m$, there exist uniquely determined $r(t), q(t) \in R$ with $\operatorname{deg}(r)<\operatorname{deg}(f)$, such that $g(t)=q(t) f(t)+r(t)$, and if $\sigma \in \operatorname{Aut}(D)$, also uniquely determined $r(t), q(t) \in R$ with $\operatorname{deg}(r)<\operatorname{deg}(f)$, such that $g(t)=f(t) q(t)+r(t)$ ([4], [35, Proposition 1]).

Let $\bmod _{r} f$ denote the remainder of right division by $f$ and $\bmod _{l} f$ the remainder of left division by $f$. The skew polynomials of degree less that $m$ canonically represent the elements of the (left resp. right) $S[t ; \sigma, \delta]$-modules $S[t ; \sigma, \delta] / S[t ; \sigma, \delta] f$ and $S[t ; \sigma, \delta] / f S[t ; \sigma, \delta]$. 
Moreover,

$$
R_{m}=\{g \in S[t ; \sigma, \delta] \mid \operatorname{deg}(g)<m\}
$$

together with the multiplication

$$
g \circ h=\left\{\begin{array}{l}
g h \text { if } \operatorname{deg}(g)+\operatorname{deg}(h)<m, \\
g h \bmod _{r} f \text { if } \operatorname{deg}(g)+\operatorname{deg}(h) \geq m,
\end{array}\right.
$$

is a unital nonassociative ring $S_{f}=\left(R_{m}, \circ\right)$ also denoted by $R / R f$.

If $\sigma \in \operatorname{Aut}(S)$, then $R_{m}$ together with

$$
g \circ h=\left\{\begin{array}{l}
g h \text { if } \operatorname{deg}(g)+\operatorname{deg}(h)<m, \\
g h \bmod _{l} f \text { if } \operatorname{deg}(g)+\operatorname{deg}(h) \geq m,
\end{array}\right.
$$

is a unital nonassociative ring ${ }_{f} S=\left(R_{m}, \circ\right)$ also denoted by $R / f R$. When the context is clear, we will drop the o notation and simply use juxtaposition for multiplication in $S_{f}$.

$S_{f}$ and ${ }_{f} S$ are unital nonassociative algebras over the commutative subring

$$
S_{0}=\left\{a \in S \mid a h=h a \text { for all } h \in S_{f}\right\}=\operatorname{Comm}\left(S_{f}\right) \cap S
$$

of $S$, and

$$
C(S) \cap \operatorname{Fix}(\sigma) \cap \operatorname{Const}(\delta) \subset S_{0} .
$$

For all invertible $a \in S$ we have $S_{f}=S_{a f}$, so that without loss of generality it suffices to only consider monic polynomials in the construction. If $f$ has degree 1 then $S_{f} \cong S$. If $f$ is reducible then $S_{f}$ contains zero divisors.

In the following, we assume $m \geq 2$ and call the algebras $S_{f}$ Petit algebras as the construction goes back to Petit [30,31] (who only considered division rings $S$ ). We will focus on the algebras $S_{f}$, since the algebras ${ }_{f} S$ are anti-isomorphic to Petit algebras [35, Proposition 3].

For $0 \neq a \in S_{f}$, left multiplication $L_{a}$ is an $S_{0}$-module endomorphism. Moreover, $R_{a}$ is a left $S$-module homomorphism for $0 \neq a \in S_{f}$.

Let $f \in S[t ; \sigma, \delta]$ have degree $m \geq 2$ and an invertible leading coefficient. Then $S_{f}$ is a free left $S$-module of rank $m$ with basis $t^{0}=1, t, \ldots, t^{m-1} . S_{f}$ is associative if and only if $R f$ is a two-sided ideal in $R$. If $S_{f}$ is not associative then $S \subset \operatorname{Nuc}_{l}\left(S_{f}\right), S \subset \operatorname{Nuc}_{m}\left(S_{f}\right)$ and

$$
\{g \in R \mid \operatorname{deg}(g)<m \text { and } f g \in R f\}=\operatorname{Nuc}_{r}\left(S_{f}\right) .
$$

When $S$ is a division ring, these inclusions become equalities. We have $t \in \mathrm{Nuc}_{r}\left(S_{f}\right)$, if and only if the powers of $t$ are associative, if and only if $t^{m} t=t t^{m}$ in $S_{f}$. If $S$ is a division ring and $S_{f}$ is not associative then $C\left(S_{f}\right)=S_{0}$. Let $f(t)=\sum_{i=0}^{m} a_{i} t^{i} \in S[t ; \sigma]$ with $a_{0}$ invertible. If the endomorphism $L_{t}$ (i.e. left multiplication by $t$ ) is surjective then $\sigma$ is surjective. In particular, if $S$ is a division ring and $f$ irreducible, then $L_{t}$ surjective implies $\sigma$ surjective. Moreover, if $\sigma$ is bijective then $L_{t}$ is surjective [35, Theorem 4].

Since $C\left(S_{f}\right)=\operatorname{Comm}\left(S_{f}\right) \cap \mathrm{Nuc}_{l}\left(S_{f}\right) \cap \mathrm{Nuc}_{m}\left(S_{f}\right) \cap \mathrm{Nuc}_{r}\left(S_{f}\right)$, we have

$$
S_{0}=\left\{a \in S \mid a h=h a \text { for all } h \in S_{f}\right\}=\operatorname{Comm}\left(S_{f}\right) \cap S \subset C\left(S_{f}\right)
$$

when $S_{f}$ is not associative. If $\operatorname{Nuc}_{l}\left(S_{f}\right)=\operatorname{Nuc}_{m}\left(S_{f}\right)=S$ this yields that the center $C\left(S_{f}\right)=\operatorname{Comm}\left(S_{f}\right) \cap S \cap \operatorname{Nuc}_{r}\left(S_{f}\right)=\operatorname{Comm}\left(S_{f}\right) \cap S$ of $S_{f}$ is identical to the ring $S_{0}$. 


\section{The Right nucleus of $S_{f}$}

In this Section, let $D$ be a division algebra with center $F, R=D[t ; \sigma, \delta]$ with $\sigma$ any endomorphism of $D$ and $\delta$ any left $\sigma$-derivation. Let $f \in R=D[t ; \sigma, \delta]$ be monic of degree $m \geq 2$ and $D_{0}=\left\{a \in D \mid a h=h a\right.$ for all $\left.h \in S_{f}\right\}$.

The largest subalgebra of $R=D[t ; \sigma, \delta]$ in which $R f$ is a two-sided ideal is the idealizer $I(f)=\{g \in R \mid f g \in R f\}$ of $R f$. The eigenring of $f$ is then defined as the quotient $E(f)=I(f) / R f=\{g \in R \mid \operatorname{deg}(g)<m$ and $f g \in R f\}$. This is also the right nucleus of the algebra $S_{f}[35$, Theorem 4].

3.1. Some general observations. The right nucleus is important when finding right factors of $f$; if $\operatorname{Nuc}_{r}\left(S_{f}\right)$ contains zero divisors then $f$ is reducible [30]. If $u, v \in \operatorname{Nuc}_{r}\left(S_{f}\right)$ are non-zero such that $u v=0$, then the greatest common right divisor $\operatorname{gcr} d(f, u)$ is a non-trivial right factor of $f$, see e.g. [35]. This was employed for instance in [17].

Moreover, if $f t \in R f$ then $t \in \operatorname{Nuc}_{r}\left(S_{f}\right)$, hence the powers of $t$ are associative in $S_{f}$. This in turn implies $t^{m} t=t t^{m}$ [35, Theorem 5]. Moreover, $f t \in R f$ if and only if $t \in \operatorname{Nuc}_{r}\left(S_{f}\right)$, if and only if the powers of $t$ are associative, if and only if $t^{m} t=t t^{m}$ [30]. This yields:

Lemma 1. Let $f \in D[t ; \sigma, \delta]$. If $t \notin \operatorname{Nuc}_{r}\left(S_{f}\right)$ or $f \in R t$ then $S_{f}$ does not have any associative subalgebra that contains all powers of $t$.

In particular, if $f$ is irreducible and $t \notin \operatorname{Nuc}_{r}\left(S_{f}\right)$, then $S_{f}$ does not have any associative subalgebra that contains all powers of $t$ (and then $f$ cannot lie in $D_{0}[t]$ ).

Proof. There exists a subset $X$ of $S_{f}$ which is a multiplicative group and contains all powers of $t$, if and only if $f t \in R f$ and $f \notin R t$ [30, (8)], i.e. if and only if $t \in \operatorname{Nuc}_{r}\left(S_{f}\right)$ and $f \notin R$. Now suppose $A$ is an associative subalgebra of $S_{f}$ that contains all powers of $t$, choose $X=A$ and obtain that $t \in \mathrm{Nuc}_{r}\left(S_{f}\right)$ and $f \notin R t$.

If $f$ is irreducible, we know that $f \notin R t$. If, additionally, $f \in D_{0}[t]$ then $D_{0}[t] /(f)$ is a subalgebra of $S_{f}$ that contains all powers of $t$, a contradiction.

Proposition 2. For all $f \in D_{0}[t], D_{0}[t] /(f)$ is a commutative subring of $S_{f}$ and

$$
D_{0}[t] /(f)=D_{0} \oplus S_{0} t \oplus \cdots \oplus S_{0} t^{m-1} \subset \operatorname{Nuc}_{r}\left(S_{f}\right) .
$$

If $\operatorname{Nuc}_{r}\left(S_{f}\right)$ is larger than $D_{0}[t] /(f)$, then $\operatorname{Nuc}_{r}\left(S_{f}\right)$ is not commutative.

Proof. $S_{f}$ contains the commutative subring $D_{0}[t] /(f)$, where $D_{0}=\operatorname{Const}(\delta) \cap C(D) \cap$ $\operatorname{Fix}(\sigma)$. This subring is isomorphic to the ring consisting of the elements $\sum_{i=0}^{m-1} a_{i} t^{i}$ with $a_{i} \in D_{0}$. In particular, we know that the powers of $t$ are associative. By Theorem [35, Theorem 4], this implies that $t \in \operatorname{Nuc}_{r}\left(S_{f}\right)$. Clearly $D_{0} \subset \operatorname{Nuc}_{r}\left(S_{f}\right)$, so if $t \in \operatorname{Nuc}_{r}\left(S_{f}\right)$ then $D_{0} \oplus D_{0} t \oplus \cdots \oplus D_{0} t^{m-1} \subset \operatorname{Nuc}_{r}\left(S_{f}\right)$, hence we obtain the assertion. The last part is trivial then.

If $f \in D_{0}[t]$ is irreducible in $D_{0}[t]$, then $D_{0}[t] /(f)$ is an algebraic field extension of $D_{0}$ of degree $m$ contained in $\mathrm{Nuc}_{r}\left(S_{f}\right)$. Thus if $K$ is a finite field, $\delta=0$ and $f$ irreducible, then $\operatorname{Nuc}_{r}\left(S_{f}\right)=F \oplus F t \oplus \cdots \oplus F t^{m-1}=F[t] /(f)$, employing the fact that in this case we know that the right nucleus has exactly $|F[t] /(f)|$ elements [24]. 
3.2. Right semi-invariant polynomials. We first investigate for which $f$ the algebra $D$ is contained in the right nucleus of $S_{f}$. By [35, Theorem 4], this implies that either $S_{f}$ is associative or $\operatorname{Nuc}\left(S_{f}\right)=D$.

Recall that $f \in R=D[t ; \sigma, \delta]$ is called right semi-invariant if for every $a \in D$ there is $b \in$ $D$ such that $f(t) a=b f(t)$ which is equivalent to $f D \subseteq D f$. Similarly, $f$ is left semi-invariant if $D f \subseteq f D[26,27]$. Moreover, $f$ is right semi-invariant if and only if $d f$ is right semiinvariant for all $d \in D^{\times}[26$, p. 8]. Hence we only need to consider monic $f$. Furthermore, if $\sigma$ is an automorphism, then $f$ is right semi-invariant if and only if it is left semi-invariant if and only if $f D=D f$ [26, Proposition 2.7]. Right semi-invariant polynomials canonically arise in the theory of semi-linear transformations [21]. For a thorough background on right semi-invariant polynomials see $[26,27]$.

If $f$ is semi-invariant and also satisfies $f(t) t=(b t+a) f(t)$ for some $a, b \in D$ then $f$ is called right invariant which is equivalent to $f R \subset R f$. If $f$ is right invariant then $R f$ is a two-sided ideal in $R$ and conversely, every two-sided ideal in $R$ is generated by a rightinvariant polynomial. That means $R$ is not simple if and only if there is a non-constant right-invariant $f \in R$. Moreover, assuming $\sigma$ is an automorphism, $R$ is not simple if and only if there is a non-constant monic semi-invariant $f \in R$ if and only if $\delta$ is a quasi-algebraic derivation [27] (this last observation actually holds for any simple ring $D$ ).

Theorem 3. $f \in R$ is right semi-invariant if and only if $D \subseteq \operatorname{Nuc}_{r}\left(S_{f}\right)$. In particular, if $f$ is right semi-invariant, then either $\operatorname{Nuc}\left(S_{f}\right)=D$ or $S_{f}$ is associative.

Proof. If $f \in R$ is right semi-invariant, $f D \subseteq D f \subseteq R f$ and hence $D \subseteq E(f)=\operatorname{Nuc}_{r}\left(S_{f}\right)$. Conversely, if $D \subseteq \operatorname{Nuc}_{r}\left(S_{f}\right)=E(f)$ then for all $d \in D$, there exists $q(t) \in R$ such that $f(t) d=q(t) f(t)$. Comparing degrees, we see $q(t) \in D$ and thus $f D \subseteq D f$.

The second assertion follows by [35, Theorem 4].

Proposition 4. $([28,(9.21)])$. Suppose $\sigma$ is an automorphism of $D$, then the following are equivalent:

(i) There exists a non-constant right semi-invariant polynomial in $R$.

(ii) $R$ is not simple.

(iii) There exist $b_{0}, \ldots, b_{n} \in D$ with $b_{n} \neq 0$ such that $b_{0} \delta_{c, \theta}+\sum_{i=1}^{n} b_{i} \delta^{i}=0$, where $\theta$ is an endomorphism of $D$ and $\delta_{c, \theta}$ denotes the $\theta$-derivation of $D$ sending $x \in D$ to $c x-\theta(x) c$.

Corollary 5. Suppose $\sigma$ is an automorphism of $D$ and $R$ is simple. Then there are no nonassociative algebras $S_{f}$ with $D \subseteq \operatorname{Nuc}_{r}\left(S_{f}\right)$. In particular, there are no nonassociative algebras $S_{f}$ with $D \subseteq \operatorname{Nuc}\left(S_{f}\right)$.

Proof. $R$ is not simple if and only if there exists a non-constant right semi-invariant polynomial in $R$ by Proposition 4, and hence the assertion follows by Theorem 3 .

Corollary 5 actually also holds when $f \in S[t ; \sigma, \delta]$, where $S$ is only a simple ring and $\sigma$ an automorphism of $S[27$, Theorem 5.2].

Recall that if $S$ is a division ring, or if $S$ is a simple ring and $\sigma \in \operatorname{Aut}(S)$, then $R=S[t ; \sigma, \delta]$ is not simple if and only if $\delta$ is quasi-algebraic [27]. Recall also that $\sigma$ is an automorphism of $D$ of finite inner order $k$ if $\sigma^{k}=I_{u}$ for some $u \in D^{\times}$. Using Theorem 3 we can rephrase 
the results [26, Lemma 2.2, Corollary 2.12, Propositions 2.3 and 2.4], [27, Corollary 2.6] on right semi-invariant polynomials in terms of the right nucleus of the nonassociative algebra $S_{f}$ :

Theorem 6. Let $f(t)=\sum_{i=0}^{m} a_{i} t^{i} \in R$ be monic of degree $m$.

(i) $D \subseteq \operatorname{Nuc}_{r}\left(S_{f}\right)$ if and only if $f(t) c=\sigma^{m}(c) f(t)$ for all $c \in D$, if and only if

$$
\sigma^{m}(c) a_{j}=\sum_{i=j}^{m} a_{i} \Delta_{i, j}(c)
$$

for all $c \in D$ and $j \in\{0, \ldots, m-1\}$.

(ii) Suppose $\sigma$ is an automorphism of $D$ of infinite inner order. Then $D \subseteq \operatorname{Nuc}_{r}\left(S_{f}\right)$ implies $S_{f}$ is associative.

(iii) Suppose $\delta=0$. Then $D \subseteq \operatorname{Nuc}_{r}\left(S_{f}\right)$ if and only if

$$
\sigma^{m}(c)=a_{j} \sigma^{j}(c) a_{j}^{-1}
$$

for all $c \in D$ and all $j \in\{0, \ldots, m-1\}$ with $a_{j} \neq 0$. Furthermore, $S_{f}$ is associative if and only if $f(t)$ satisfies (2) and $f(t) \in \operatorname{Fix}(\sigma)[t] \subset \operatorname{Fix}(\sigma)[t ; \sigma]$.

(iv) Suppose $\sigma=i d$. Then $D \subseteq \operatorname{Nuc}_{r}\left(S_{f}\right)$ is equivalent to

$$
c a_{j}=\sum_{i=j}^{m}\left(\begin{array}{l}
i \\
j
\end{array}\right) a_{i} \delta^{i-j}(c)
$$

for all $c \in D, j \in\{0, \ldots, m-1\}$. Furthermore, $S_{f}$ is associative if and only if $f(t)$ satisfies (3) and $f(t) \in \operatorname{Const}(\delta)[t] \subset \operatorname{Const}(\delta)[t ; \delta]$.

(v) Suppose $\delta=0$ and $\sigma$ is an automorphism of $D$ of finite inner order $k$, i.e. $\sigma^{k}=I_{u}$ for some $u \in D^{\times}$. Then the polynomials $g \in D[t ; \sigma]$ such that $D \subseteq \operatorname{Nuc}_{r}\left(S_{g}\right)$ are precisely those of the form

$$
g(t)=b \sum_{j=0}^{n} c_{j} u^{n-j} t^{j k}
$$

where $n \in \mathbb{N}, c_{n}=1, c_{j} \in F$ and $b \in D^{\times}$. Furthermore, $S_{g}$ is associative if and only if $g(t)$ has the form (4) and $g(t) \in \operatorname{Fix}(\sigma)[t] \subset \operatorname{Fix}(\sigma)[t ; \sigma]$.

3.3. Right $B$-weak semi-invariant polynomials. Let now $B$ be a subring of $D$. We can find conditions on $f$ such that $B$ is contained in $\operatorname{Nuc}_{r}\left(S_{f}\right)$ by generalizing the definition of right semi-invariant polynomials as follows: we say $f \in D[t ; \sigma, \delta]$ is (right) $B$-weak semiinvariant if $f B \subseteq D f$. Clearly any right semi-invariant polynomial is also $B$-weak semiinvariant for every subring $B$ of $D$. We call $f \in R$ a (right) $B$-weak invariant polynomial if $f$ is right $B$-weak semi-invariant and $f(t) t=(b t+a) f(t)$ for some $a, b \in B$.

Note that when we have an extension of rings $B \subset D$, which induces an extension of skewpolynomial rings $B[t, \sigma, \delta] \subset D[t, \sigma, \delta]$ (i.e., $\left.\sigma\right|_{B}=\sigma,\left.\delta\right|_{B}=\delta$ ), every right semi-invariant $f \in B[t, \sigma, \delta]$ is right $B$-weak semi-invariant in $D[t, \sigma, \delta]$, and every invariant $f \in B[t, \sigma, \delta]$ is right $B$-weak invariant in $D[t, \sigma, \delta]$. 
Example 7. Let $K$ be a field, $\sigma$ be a non-trivial automorphism of $K, L=\operatorname{Fix}\left(\sigma^{j}\right)$ be the fixed field of $\sigma^{j}$ for some $j>1$ and $f(t)=\sum_{i=0}^{n} a_{i} t^{i j} \in K[t ; \sigma]$. Then

$$
f(t) l=\sum_{i=0}^{n} a_{i} t^{i j} l=\sum_{i=0}^{n} a_{i} \sigma^{i j}(l) t^{i j}=\sum_{i=0}^{n} a_{i} l t^{i j}=l f(t),
$$

for all $l \in L$ and hence $f L \subseteq L f$. In particular, $f$ is $L$-weak semi-invariant.

Proposition 8. Let $B$ be a subring of $D$.

(i) $f$ is $B$-weak semi-invariant if and only if $B \subseteq \operatorname{Nuc}_{r}\left(S_{f}\right)$.

(ii) If $f$ is $B$-weak semi-invariant but not right invariant, then $B \subseteq \operatorname{Nuc}\left(S_{f}\right) \subseteq D$.

Proof. (i) If $f \in R$ is $B$-weak semi-invariant, $f B \subseteq D f \subseteq R f$ and hence $B \subseteq \operatorname{Nuc}_{r}\left(S_{f}\right)$. Conversely, if $B \subseteq \operatorname{Nuc}_{r}\left(S_{f}\right)$ then for all $b \in B$, there exists $q(t) \in R$ such that $f(t) b=$ $q(t) f(t)$. Comparing degrees, we see $q(t) \in D$ and thus $f B \subseteq D f$.

(ii) If $f$ is $B$-weak semi-invariant but not right invariant, the assertion follows from (i) and [35, Theorem 4].

Proposition 9. Let $B$ be a subring of $D$ and $f \in R$ be a right $B$-weak invariant polynomial. Then

$$
B \oplus B t \oplus \cdots \oplus B t^{m-1} \subset \operatorname{Nuc}_{r}\left(S_{f}\right) .
$$

Proof. (i) If $f \in R$ is a right $B$-weak invariant polynomial then $B \subset \operatorname{Nuc}_{r}\left(S_{f}\right)$ by Proposition 8. Since $f(t) t=(b t+a) f(t)$ for some $a, b \in B$, we have $f t \in R f$ which implies $t \in \operatorname{Nuc}_{r}\left(S_{f}\right)$, hence the powers of $t$ are associative. This in turn implies $t^{m} t=t t^{m}$ ([35, Theorem 5] and [30]). Now $\operatorname{Nuc}_{r}\left(S_{f}\right)$ is an associative subalgebra of $S_{f}$, thus $B \oplus B t \oplus \cdots \oplus B t^{m-1} \subset$ $\operatorname{Nuc}_{r}\left(S_{f}\right)$.

We then obtain results similar to Theorem 6 (i), (iii) and (v) for $B$-weak semi-invariant polynomials:

Proposition 10. Let $f(t)=\sum_{i=0}^{m} a_{i} t^{i} \in D[t ; \sigma, \delta]$ be monic of degree $m$ and $B$ a subring of $D$.

(i) $f$ is B-weak semi-invariant if and only if $f(t) c=\sigma^{m}(c) f(t)$ for all $c \in B$, if and only if

$$
\sigma^{m}(c) a_{j}=\sum_{i=j}^{m} a_{i} \Delta_{i, j}(c)
$$

for all $c \in B, j \in\{0, \ldots, m-1\}$.

(ii) Suppose $\delta=0$. Then $f$ is $B$-weak semi-invariant if and only if $\sigma^{m}(c) a_{j}=a_{j} \sigma^{j}(c)$ for all $c \in B, j \in\{0, \ldots, m-1\}$.

(iii) Suppose $\sigma=i d$. Then $f$ is $B$-weak semi-invariant if and only if

$$
c a_{j}=\sum_{i=j}^{m}\left(\begin{array}{l}
i \\
j
\end{array}\right) a_{i} \delta^{i-j}(c)
$$

for all $c \in B, j \in\{0, \ldots, m-1\}$. 
Proof. (i) We have

$$
f(t) c=\sum_{i=0}^{m} a_{i} t^{i} c=\sum_{i=0}^{m} a_{i} \sum_{j=0}^{i} \Delta_{i, j}(c) t^{j}=\sum_{j=0}^{m} \sum_{i=j}^{m} a_{i} \Delta_{i, j}(c) t^{j}
$$

for all $c \in B$, hence the $t^{m}$ coefficient of $f(t) c$ is $\Delta_{m, m}(c)=\sigma^{m}(c)$, and so $f$ is $B$-weak semi-invariant if and only if $f(t) c=\sigma^{m}(c) f(t)$ for all $c \in B$. Comparing the $t^{j}$ coefficient of (7) and $\sigma^{m}(c) f(t)$ for all $j \in\{0, \ldots, m-1\}$ yields (5).

(ii) When $\delta=0, \Delta_{i, j}=0$ unless $i=j$ in which case $\Delta_{j, j}=\sigma^{j}$. Therefore (5) simplifies to $\sigma^{m}(c) a_{j}=a_{j} \sigma^{j}(c)$ for all $c \in B, j \in\{0, \ldots, m-1\}$.

(iii) When $\sigma=i d$ we have

$$
t^{i} c=\sum_{j=0}^{i}\left(\begin{array}{l}
i \\
j
\end{array}\right) \delta^{i-j}(c)
$$

for all $c \in D$ by $[20,(1.1 .26)]$ and thus

$$
f(t) c=\sum_{i=0}^{m} a_{i} t^{i} c=\sum_{i=0}^{m} a_{i} \sum_{j=0}^{i}\left(\begin{array}{l}
i \\
j
\end{array}\right) \delta^{i-j}(c) t^{j}=\sum_{j=0}^{m} \sum_{i=j}^{m}\left(\begin{array}{l}
i \\
j
\end{array}\right) a_{i} \delta^{i-j}(c) t^{j}
$$

for all $c \in B$. Furthermore $f$ is $B$-weak semi-invariant is equivalent to $f(t) c=c f(t)$ for all $c \in B$ by (i). Comparing the $t^{j}$ coefficient of (8) and $c f(t)=\sum_{i=0}^{m} c a_{i} t^{i}$ for all $c \in B$, $j \in\{0, \ldots, m-1\}$ yields $(6)$.

\section{4. (Right) Division algebras obtained from Petit algebras}

Petit algebras can be used to find classes of algebras that are right but not left division algebras.

Let $D$ be a division algebra with center $F$ and $R=D[t ; \sigma, \delta]$. We say $f \in R$ is bounded if there exists $0 \neq f^{*} \in R$ such that $R f^{*}=f^{*} R$ is the largest two-sided ideal of $R$ contained in $R f$. The element $f^{*}$ is determined by $f$ up to multiplication on the left by elements of $D^{\times}$. If $f \in R$ is irreducible then $E(f)$ is a division ring [30, p. 13-07].

Remark 11. If $\sigma$ is an automorphism and $f$ is bounded, then $f$ is irreducible if and only if $E(f)=\operatorname{Nuc}_{r}\left(S_{f}\right)$ is an associative division algebra [15, Proposition 4] which sums up classical results from [22]. The condition that $f$ is bounded is necessary here, as is shown in $[15$, Example 3] where $f \in \mathbb{Q}(x)[t ; d / d t]$ is reducible in the differential operator ring $\mathbb{Q}(x)[t ; d / d t]$, but $\operatorname{Nuc}_{r}\left(S_{f}\right)$ is a division algebra. For instance, if $D$ is a finite field and $\delta=0$, all polynomials are bounded and hence $f$ is irreducible if and only if $E(f)$ is a finite field [13, Theorem 3.3].

The argument leading up to [30, Section 2., (6)] implies that $S_{f}$ has no zero divisors if and only if $f$ is irreducible, which is in turn equivalent to $S_{f}$ being a right division ring (i.e., right multiplication $R_{a}$ in $S_{f}$ is bijective for all $0 \neq a \in S_{f}$ ):

Theorem 12. ([30, (6)], but without a full proof). Let $f \in R$ have degree $m$ and $0 \neq a \in S_{f}$. (i) $R_{a}$ is bijective is equivalent to 1 being a greatest common right divisor of $f$ and a (i.e., $D a(t)+D f(t)=D)$.

(ii) $S_{f}$ is a right division algebra if and only if $f$ is irreducible, if and only if $S_{f}$ has no zero 
divisors.

(iii) If $f$ is irreducible then $L_{a}$ is injective for all $0 \neq a \in S_{f}$.

Proof. (i) Let $0 \neq a \in S_{f}$. Since $S_{f}$ is a free left $D$-module of finite rank $m$ and $R_{a}$ is left $D$-linear, $R_{a}$ is bijective if and only if it is injective [19, Chapter IV, Corollary 2.14], which is equivalent to $\operatorname{ker}\left(R_{a}\right)=\{0\}$. Now $R_{a}(z)=z a=0$ is equivalent to $z a \in R f$, which means we can write

$$
\operatorname{ker}\left(R_{a}\right)=\left\{z \in R_{m} \mid z a \in R f\right\}
$$

Furthermore, $R$ is a left principal ideal domain, which implies $z a \in R f$ if and only if $z a \in R a \cap R f=R g=R h a$, where $g=h a$ is the least common left multiple of $a$ and $f$. Therefore $z a \in R f$ is equivalent to $z \in R h$, and hence $\operatorname{ker}\left(R_{a}\right) \neq\{0\}$, if and only if there exists a polynomial of degree strictly less than $m$ in $R h$, which is equivalent to $\operatorname{deg}(h) \leq m-1$.

Let $b \in R$ be a right greatest common divisor of $a$ and $f$. Then $\operatorname{deg}(f)+\operatorname{deg}(a)=$ $\operatorname{deg}(g)+\operatorname{deg}(b)=\operatorname{deg}(h a)+\operatorname{deg}(b)$ by [20, Proposition 1.3.1], and so $\operatorname{deg}(b)=\operatorname{deg}(f)-\operatorname{deg}(h)$. Thus $\operatorname{deg}(h) \leq m-1$ if and only if $\operatorname{deg}(b) \geq 1$. We conclude $\operatorname{ker}\left(R_{a}\right)=\{0\}$ if and only if $\operatorname{deg}(b)=0$, if and only if 1 is a right greatest common divisor of $f(t)$ and $a$. In particular, $S_{f}$ is a right division algebra if and only if $R_{a}$ is bijective for all $0 \neq a \in S_{f}$, if and only if 1 is a right greatest common divisor of $f(t)$ and $a$ for all $0 \neq a \in S_{f}$, if and only if $f(t)$ is irreducible.

(ii) If $f$ is irreducible then $L_{a}$ and $R_{a}$ are injective for all $0 \neq a \in S_{f}$ (i), therefore $S_{f}$ has no zero divisors. The converse of the last equivalence statement is trivial.

(iii) If $R_{h}$ is bijective this automatically implies that $L_{h}$ is injective, for all $0 \neq h \in S_{f}$.

Lemma 13. If $f \in R$ is right invariant, then $S_{f}$ is associative and a division algebra if and only if $f$ is irreducible.

Proof. Suppose $f$ is right invariant. Then $S_{f}$ is associative by [35, Theorem 4]. If $f$ is reducible then $S_{f}$ is trivially not a division algebra. Conversely, if $f$ is irreducible the maps $R_{b}$ are bijective for all $0 \neq b \in S_{f}$ by Theorem 12. This implies the maps $L_{b}$ are also bijective for all $0 \neq b \in S_{f}$ by [7, Lemma 1B], and so $S_{f}$ is a division algebra.

This implies a generalization of Theorem [35, Theorem 4]:

Theorem 14. Let $f(t)=\sum_{i=0}^{m} a_{i} t^{i} \in D[t ; \sigma]$ be monic and $a_{0} \neq 0$. Then for every $j \in\{1, \ldots, m-1\}, L_{t^{j}}$ is surjective if and only if $\sigma$ is surjective. In particular, if $\sigma$ is not surjective then $S_{f}$ is not a left division algebra.

Proof. We first prove the result for $j=1$ : Given $z=\sum_{i=0}^{m-1} z_{i} t^{i} \in S_{f}$, we have

$$
\begin{aligned}
L_{t}(z) & =t \circ z=\sum_{i=0}^{m-2} \sigma\left(z_{i}\right) t^{i+1}+\sigma\left(z_{m-1}\right) t \circ t^{m-1} \\
& =\sum_{i=1}^{m-1} \sigma\left(z_{i-1}\right) t^{i}+\sigma\left(z_{m-1}\right) \sum_{i=0}^{m-1} a_{i} t^{i} .
\end{aligned}
$$

$\Rightarrow$ : Suppose $L_{t}$ is surjective, then given any $b \in D$ there exists $z \in S_{f}$ such that $t \circ z=b$. The $t^{0}$-coefficient of $L_{t}(z)$ is $\sigma\left(z_{m-1}\right) a_{0}$ by (9), and thus for all $b \in D$ there exists $z_{m-1} \in D$ 
such that $\sigma\left(z_{m-1}\right) a_{0}=b$. Therefore $\sigma$ is surjective.

$\Leftarrow$ : Suppose $\sigma$ is surjective and let $g=\sum_{i=0}^{m-1} g_{i} t^{i} \in S_{f}$. Define

$$
z_{m-1}=\sigma^{-1}\left(g_{0} a_{0}^{-1}\right), z_{i-1}=\sigma^{-1}\left(g_{i}\right)-z_{m-1} \sigma^{-1}\left(a_{i}\right)
$$

for all $i \in\{1, \ldots, m-1\}$. Then

$$
L_{t}(z)=\sigma\left(z_{m-1}\right) a_{0}+\sum_{i=1}^{m-1}\left(\sigma\left(z_{i-1}\right)+\sigma\left(z_{m-1}\right) a_{i}\right) t^{i}=\sum_{i=0}^{m-1} g_{i} t^{i}=g,
$$

by (9), which implies $L_{t}$ is surjective.

Hence $L_{t}$ surjective is equivalent to $\sigma$ surjective. To prove the result for all $j \in\{1, \ldots, m-$ 1 ) we show that

$$
L_{t^{j}}=L_{t}^{j},
$$

for all $j \in\{1, \ldots, m-1\}$, then it follows $\sigma$ is surjective if and only if $L_{t}$ is surjective if and only if $L_{t}^{j}=L_{t^{j}}$ is surjective. In the special case when $D=\mathbb{F}_{q}$ is a finite field, $\sigma$ is an automorphism and $f$ is monic and irreducible, the equality (10) is proven in [24, p. 12]. A similar proof also works in our context: suppose inductively that $L_{t^{j}}=L_{t}^{j}$ for some $j \in\{1, \ldots, m-2\}$. Then $L_{t}^{j}(b)=t^{j} b \bmod _{r} f$ for all $b \in R_{m}$. Let $L_{t}^{j}(b)=b^{\prime}$ so that $t^{j} b=q f+b^{\prime}$ for some $q \in R$. We have

$$
\begin{aligned}
L_{t}^{j+1}(b) & =L_{t}\left(L_{t}^{j}(b)\right)=L_{t}\left(b^{\prime}\right)=L_{t}\left(t^{j} b-q f\right)=t \circ\left(t^{j} b-q f\right) \\
& =\left(t^{j+1} b-t q f\right) \bmod _{r} f=t^{j+1} b \bmod _{r} f=L_{t^{j+1}}(b),
\end{aligned}
$$

hence (10) follows by induction.

Recall that for $\delta=0, L_{t}$ is a pseudo-linear transformation, i.e. $L_{t}(a h(t))=\sigma(a) L_{t}(h(t))$ for all $a \in S, h(t) \in S_{f}$, and that $L_{h}=h(t)\left(L_{t}\right)=\sum_{i=0}^{m-1} a_{i} L_{t}^{i}$ for $h(t)=\sum_{i=0}^{m-1} a_{i} t^{i}$. If $f$ is irreducible, then $L_{t}$ is irreducible, that means the only $L_{t}$-invariant subspaces of the left $D$-module $D^{m}$ are $\{0\}$ and $D^{m}$, as pointed out in [24].

Corollary 15. Suppose $\sigma$ is not surjective and $f \in D[t ; \sigma]$ is irreducible. Then $S_{f}$ has no zero divisors and is a right division algebra but not a left division algebra. In particular, $S_{f}$ is an infinite-dimensional $D_{0}$-algebra.

The following result was stated but not proved by Petit [30, (7)]:

Theorem 16. Let $f \in D[t ; \sigma, \delta]$ be such that $S_{f}$ is either a finite-dimensional $D_{0}$-vector space or a right $\operatorname{Nuc}_{r}\left(S_{f}\right)$-module which is free of finite rank. Then $S_{f}$ is a division algebra if and only if $f$ is irreducible.

Proof. When $S_{f}$ is associative the assertion follows by Lemma 13 so suppose $S_{f}$ is not associative. If $f$ is reducible, $S_{f}$ is not a division algebra. Conversely, suppose $f$ is irreducible, so that $S_{f}$ is a right division algebra by Theorem 12 . Let $0 \neq a \in S_{f}$ be arbitrary, then $L_{a}$ is injective for all $0 \neq a \in S_{f}$ by Lemma 12 . We prove $L_{a}$ is surjective, hence that $S_{f}$ is also a left division algebra:

(i) If $S_{f}$ is a finite-dimensional $D_{0}$-vector space then $L_{a}$ is clearly surjective by [19, Chapter IV, Corollary 2.14], since $L_{a}$ is $F$-linear. 
(ii) Suppose $S_{f}$ is a free right $\operatorname{Nuc}_{r}\left(S_{f}\right)$-module of finite rank, then $E(f)$ is a division ring. Furthermore, $L_{a}$ is right $\operatorname{Nuc}_{r}\left(S_{f}\right)$-linear. Therefore $L_{a}$ is again surjective by [19, Chapter IV, Corollary 2.14].

Theorem 17. Let $\sigma$ be an automorphism of $D, B$ be a subring of $D$ such that $D$ is a free right $B$-module of finite rank, and $f \in D[t ; \sigma, \delta]$ be $B$-weak semi-invariant. Then $S_{f}$ is a division algebra if and only if $f$ is irreducible. In particular, if $\sigma$ is an automorphism of $D$ and $f$ is right semi-invariant then $S_{f}$ is a division algebra if and only if $f$ is irreducible.

Proof. If $f$ is reducible then $S_{f}$ is not a division algebra. Conversely, suppose $f$ is irreducible. Then $S_{f}$ is a right division algebra by Theorem 12 so we are left to show $S_{f}$ is also a left division algebra. Let $0 \neq a \in S_{f}$ be arbitrary and recall $L_{a}$ is injective by Lemma 12 . Since $f$ is $B$-weak semi-invariant, $B \subseteq \operatorname{Nuc}_{r}\left(S_{f}\right)$ which implies that $L_{a}$ is right $B$-linear. $S_{f}$ is a free right $D$-module of rank $m=\operatorname{deg}(f)$ because $\sigma$ is an automorphism. Since $D$ is a free right $B$-module of finite rank then also $S_{f}$ is a free right $B$-module of finite rank. Thus $[19$, Chapter IV, Corollary 2.14] implies $L_{a}$ is bijective as required.

Theorem 18. Let $f \in R=D[t ; \sigma, \delta]$ be irreducible. Then $f$ is bounded if and only if $S_{f}$ is free of finite rank as a $\operatorname{Nuc}_{r}\left(S_{f}\right)$-module. In this case, $S_{f}$ is a division algebra.

Proof. The first part of the statement is [8, Theorem 4]. Since $f$ irreducible, $S_{f}$ is a right division algebra and $L_{a}$ is injective for all $0 \neq a \in S_{f}$ as observed in [30, Section 2., (7)]. The second part then follows from the fact that $S_{f}$ is free of finite rank as a $\operatorname{Nuc}_{r}\left(S_{f}\right)$-module, which means the injective $\operatorname{Nuc}_{r}\left(S_{f}\right)$-linear map $L_{a}$ is also surjective.

For $\sigma=0$ this is [37, Theorem 2].

Corollary 19. Let $f \in R=D[t ; \sigma, \delta]$ be irreducible.

(i) Let $\sigma$ be surjective and $D=\operatorname{Nuc}_{r}\left(S_{f}\right)$. Then $f$ is bounded and $S_{f}$ is a division algebra.

(ii) Let $f$ be bounded, then $S_{f}$ is a division algebra.

Proof. (i) If $\sigma$ is surjective then $S_{f}$ is a right $D$-module, free of rank $m$. Since $D=\operatorname{Nuc}_{r}\left(S_{f}\right)$, Theorem 18 yields the assertion.

(ii) is trivial.

If $\sigma$ is an automorphism, $R=D[t ; \sigma, \delta]$ has finite rank over its center if and only if $D$ is of finite rank over $C_{t}=\{a \in F \mid a t=t a\}$ if and only if all polynomials of $R$ are bounded and if for all $f$ of degree non-zero, $\operatorname{deg}\left(f^{*}\right) / \operatorname{deg}(f)$ is bounded in $\mathbb{Q}\left(f^{*}\right.$ being the bound of f) $\left[9\right.$, Theorem IV]. Since $C_{t}=\operatorname{Const}(\delta) \cap \operatorname{Fix}(\sigma)=D_{0} \subset F$ we conclude:

Proposition 20. Assume $R=D[t ; \sigma, \delta], \sigma$ is an automorphism, and one of the two following equivalent conditions hold:

(i) $R=D[t ; \sigma, \delta]$ has finite rank over its center;

(ii) $D$ has finite rank over $D_{0}$.

Then every $f \in R$ is bounded. In particular, if $f$ is irreducible then $S_{f}$ is a division algebra.

For $\sigma=0$, this is [37, Proposition 3]. 
Suppose now that $\sigma$ is an automorphism. Then $S_{f}$ is a free right $D$-module of rank $m$ and since $L_{a}$ is $\operatorname{Nuc}_{r}\left(S_{f}\right)$-linear for any non-zero $a \in S_{f}$, in this case $S_{f}$ is a division algebra for an irreducible $f$ if $D \subset \operatorname{Nuc}_{r}\left(S_{f}\right)$ or if there is a subalgebra $B \subset D$ such that $B \subset \operatorname{Nuc}_{r}\left(S_{f}\right)$ and $D$ has finite rank as a right $B$-module (these conditions were not stated in [30, p. 13-14] but seem necessary). We obtain:

Proposition 21. Suppose that $\sigma$ is an automorphism and $f$ is irreducible.

(i) If $D \subset \mathrm{Nuc}_{r}\left(S_{f}\right)$ then $S_{f}$ is a division algebra.

(ii) If there is a subalgebra $B \subset D$ such that $B \subset \operatorname{Nuc}_{r}\left(S_{f}\right)$ and $D$ is free of finite rank as a right $B$-module then $S_{f}$ is a division algebra.

Proof. $S_{f}$ is a right $D$-module and left multiplication $L_{a}$ is $\operatorname{Nuc}_{r}\left(S_{f}\right)$-linear, so in particular $D$-linear. Since $f$ is irreducible, $L_{a}$ is injective for all nonzero $a \in S_{f}$. If $D \subset \operatorname{Nuc}_{r}\left(S_{f}\right)$ then $S_{f}$ is a free right $D$-module of rank $m$, and if there is a subalgebra $B \subset D$ such that $B \subset \operatorname{Nuc}_{r}\left(S_{f}\right)$ and $S$ is free of finite rank as a right $B$-module, then $S_{f}$ is a free right $B$-module of finite rank. Thus $L_{a}$ is bijective for all nonzero $a \in S_{f}$.

\section{IrReducibility CRITERIA For SOME POLYNOMIALS In $R=D[t ; \sigma]$}

Let $D$ be a division algebra over $F$ and $f(t)=t^{m}-\sum_{i=0}^{m-1} a_{i} t^{i} \in R=D[t ; \sigma]$.

5.1. There are already several irreducibility criteria for $f$ available in the literature. We start by collecting some that are useful for constructing (right) division algebras $S_{f}$ for the convenience of the reader.

We first determine the remainder after dividing $f(t)$ on the right by $t-b$ where $b \in D$. By $[20$, p. $15 \mathrm{ff}]$ we have $\left.(t-b)\right|_{r} f(t)$ is equivalent to

$$
a_{m} N_{m}(b)-\sum_{i=0}^{m-1} a_{i} N_{i}(b)=0
$$

where $N_{i}(b)=\sigma^{i-1}(b) \cdots \sigma(b) b$ for $i>0$ and $N_{0}(b)=1$, i.e. to this remainder being zero.

When $\sigma$ is an automorphism of $D$, we can also determine the remainder after dividing $f(t)$ on the left by $(t-b), b \in D$ : Similarly to $[20$, p. $15 \mathrm{ff}]$ we have

$$
\begin{aligned}
t^{i}-b \sigma^{-1}(b) & \cdots \sigma^{1-i}(b)=(t-b)\left(t^{i-1}+\sigma^{-1}(b) t^{i-2}+\right. \\
\sigma^{-1}(b) \sigma^{-2}(b) t^{i-3}+\ldots+\sigma^{-1}(b) \sigma^{-2}(b) & \left.\cdots \sigma^{1-i}(b)\right)
\end{aligned}
$$

for all $i \in \mathbb{N}$. Multiplying (12) on the right by $\sigma^{-i}\left(a_{i}\right)$, and using $a_{i} t^{i}=t^{i} \sigma^{-i}\left(a_{i}\right)$ gives

$$
\begin{aligned}
& a_{i} t^{i}-b \sigma^{-1}(b) \cdots \sigma^{1-i}(b) \sigma^{-i}\left(a_{i}\right) \\
& =(t-b)\left(t^{i-1}+\sigma^{-1}(b) t^{i-2}+\ldots+\sigma^{-1}(b) \sigma^{-2}(b) \cdots \sigma^{1-i}(b)\right) \sigma^{-i}\left(a_{i}\right) .
\end{aligned}
$$

Summing over $i$, we obtain

$$
f(t)=(t-b) q(t)+M_{m}(b)-\sum_{i=0}^{m-1} M_{i}(b) \sigma^{-i}\left(a_{i}\right)
$$

for some $q(t) \in R$ where $M_{0}(b)=1, M_{1}(b)=b$ and $M_{i}(b)=b \sigma^{-1}(b) \cdots \sigma^{1-i}(b)$ for $i \geq 2$. We immediately conclude: 
Proposition 22. Suppose $\sigma$ is an automorphism. Then $\left.(t-b)\right|_{l} f(t)$ if and only if $M_{m}(b)-$ $\sum_{i=0}^{m-1} M_{i}(b) \sigma^{-i}\left(a_{i}\right)=0$.

Corollary 23. Suppose $\sigma$ is an automorphism and $f(t)=t^{m}-a \in D[t ; \sigma]$. Then $f(t)$ has a left linear divisor if and only if it has a right linear divisor.

Proof. Let $b \in D$, then $\left.(t-b)\right|_{r} f(t)$ is equivalent to $\sigma^{m-1}(b) \cdots \sigma(b) b=a$ by (11), if and only if $c \sigma^{-1}(c) \cdots \sigma^{1-m}(c)=a$ where $c=\sigma^{m-1}(b)$, if and only if $\left.(t-c)\right|_{l} f(t)$ by Proposition 22 .

Theorem 24. [30, (17), (18)] (i) $f(t)=t^{2}-a_{1} t-a_{0} \in D[t ; \sigma]$ is irreducible if and only if

$$
\sigma(b) b-a_{1} b-a_{0} \neq 0
$$

for all $b \in D$.

(ii) Suppose $\sigma$ is an automorphism. $f(t)=t^{3}-a_{2} t^{2}-a_{1} t-a_{0} \in D[t ; \sigma]$ is irreducible if and only if

$$
\sigma^{2}(b) \sigma(b) b-\sigma^{2}(b) \sigma(b) a_{2}-\sigma^{2}(b) \sigma\left(a_{1}\right)-\sigma^{2}\left(a_{0}\right) \neq 0
$$

and

$$
\sigma^{2}(b) \sigma(b) b-a_{2} \sigma(b) b-a_{1} b-a_{0} \neq 0
$$

for all $b \in D$.

Corollary 25. Suppose $\sigma$ is an automorphism, then $f(t)=t^{3}-a \in D[t ; \sigma]$ is irreducible if and only if $\sigma^{2}(b) \sigma(b) b \neq a$ for all $b \in D$.

Proof. By Corollary 23, $f(t)$ has a right linear divisor if and only if it has a left linear divisor. Therefore $f(t)$ is irreducible if and only if $(t-b) \nmid_{r} f(t)$ for all $b \in D$, if and only if $\sigma^{2}(b) \sigma(b) b \neq a$ for all $b \in D$ by $(11)$.

Lemma 26. Let $f(t) \in R=D[t ; \sigma]$ and suppose $f(t)=q(t) g(t)$ for some $q(t), g(t) \in R$. Then $f(b t)=q(b t) g(b t)$ for all $b \in D_{0}=F \cap \operatorname{Fix}(\sigma)$.

Proof. Write $q(t)=\sum_{i=0}^{l} q_{i} t^{i}, g(t)=\sum_{j=0}^{n} g_{j} t^{j}$, then

$$
f(t)=q(t) g(t)=\sum_{i=0}^{l} \sum_{j=0}^{n} q_{i} t^{i} g_{j} t^{j}=\sum_{i=0}^{l} \sum_{j=0}^{n} q_{i} \sigma^{i}\left(g_{j}\right) t^{i+j},
$$

and so we obtain for all $b \in D_{0}$ :

$$
\begin{aligned}
q(b t) g(b t) & =\sum_{i=0}^{l} q_{i}(b t)^{i} \sum_{j=0}^{n} g_{j}(b t)^{j}=\sum_{i=0}^{l} \sum_{j=0}^{n} q_{i} \sigma^{i}\left(g_{j}\right) b^{i+j} t^{i+j} \\
& =\sum_{i=0}^{l} \sum_{j=0}^{n} q_{i} \sigma^{i}\left(g_{j}\right)(b t)^{i+j}=f(b t) .
\end{aligned}
$$

Theorem 27. [3, p. 344] Let $\sigma$ be an endomorphism of $D, f(t)=t^{m}-a \in R=D[t ; \sigma]$ and suppose $D_{0}=F \cap \operatorname{Fix}(\sigma)$ contains a primitive mth root of unity $\omega$. If $g(t) \in R$ is a monic irreducible polynomial dividing $f(t)$ on the right, then the degree $d$ of $g(t)$ divides $m$ and $f(t)$ is the product of $m / d$ polynomials of degree $d$. 
For a proof of Theorem 27 see [4]; the special case where $\sigma$ is an automorphism of order $m$ is shown in [12, Proposition 3.7.5].

Theorem 27 implies [1, Lemma 10], cf. also [36, Theorem 6 (iii)], which improves [30, (19)]:

Theorem 28. Suppose $m$ is prime, $\sigma$ is an endomorphism of $D$ and $D_{0}=F \cap \operatorname{Fix}(\sigma)$ contains a primitive $m$ th root of unity. Then $f(t)=t^{m}-a \in D[t ; \sigma]$ is irreducible if and only if it has no right linear divisors, if and only if

$$
a \neq \sigma^{m-1}(b) \cdots \sigma(b) b
$$

for all $b \in D$.

Proof. Let $g(t) \in D[t ; \sigma]$ be an irreducible polynomial of degree $d$ dividing $f(t)$ on the right. Without loss of generality $g(t)$ is monic, otherwise if $g(t)$ has leading coefficient $c \in D^{\times}$, then $c^{-1} g(t)$ is monic and also right divides $f(t)$. Thus $d$ divides $m$ by Theorem 27 and since $m$ is prime, either $d=m$, in which case $g(t)=f(t)$, or $d=1$, which means $f(t)$ can be written as a product of $m$ linear factors. Therefore $f(t)$ is irreducible if and only if $(t-b) \nmid_{r} f(t)$ for all $b \in D$, if and only if $a \neq \sigma^{m-1}(b) \cdots \sigma(b) b$, for all $b \in D$ by (11).

5.2. Skew polynomials of degree four. Suppose $\sigma$ is an automorphism of $D$ and $f(t)=$ $t^{4}-a_{3} t^{3}-a_{2} t^{2}-a_{1} t-a_{0} \in R=D[t ; \sigma]$. Then either $f(t)$ is irreducible, $f(t)$ is divisible by a linear factor from the right, from the left, or $f(t)=g(t) h(t)$ for some $g(t), h(t) \in R$ of degree 2. In (11) and Proposition 22 we computed the remainders after dividing $f(t)$ by a linear polynomial on the right and the left. We now compute the remainder after dividing $f(t)$ by $t^{2}-c t-d$ on the right, with $c, d \in D$. To do this we use the identities

$$
\begin{gathered}
t^{2}=\left(t^{2}-c t-d\right)+(c t+d), \\
t^{3}=(t+\sigma(c))\left(t^{2}-c t-d\right)+(\sigma(d)+\sigma(c) c) t+\sigma(c) d,
\end{gathered}
$$

and

$$
\begin{aligned}
t^{4} & =\left(t^{2}+\sigma^{2}(c) t+\sigma^{2}(d)+\sigma^{2}(c) \sigma(c)\right)\left(t^{2}-c t-d\right) \\
& +\left(\sigma^{2}(c) \sigma(c) c+\sigma^{2}(d) c+\sigma^{2}(c) \sigma(d)\right) t+\sigma^{2}(d) d+\sigma^{2}(c) \sigma(c) d .
\end{aligned}
$$

If we define

$$
\begin{gathered}
M_{0}(c, d)(t)=1, M_{1}(c, d)(t)=t, M_{2}(c, d)(t)=c t+d \\
M_{3}(c, d)(t)=(\sigma(d)+\sigma(c) c) t+\sigma(c) d, \\
M_{4}(c, d)(t)=\left(\sigma^{2}(c) \sigma(c) c+\sigma^{2}(d) c+\sigma^{2}(c) \sigma(d)\right) t+\sigma^{2}(d) d+\sigma^{2}(c) \sigma(c) d,
\end{gathered}
$$

then multiplying (13), (14) and (15) on the left by $a_{i}$ and summing over $i$ yields

$$
f(t)=q(t)\left(t^{2}-c t-d\right)+M_{4}(c, d)(t)-\sum_{i=0}^{3} a_{i} M_{i}(c, d)(t)
$$

for some $q(t) \in R$. Thus the remainder after dividing $f(t)$ on the right by $t^{2}-c t-d$ is

$$
M_{4}(c, d)(t)-\sum_{i=0}^{3} a_{i} M_{i}(c, d)(t)
$$

which evidently implies: 
Proposition 29. $f(t)=t^{4}-a_{3} t^{3}-a_{2} t^{2}-a_{1} t-a_{0} \in R=D[t ; \sigma] .\left.\left(t^{2}-c t-d\right)\right|_{r} f(t)$ is equivalent to

$$
\sigma^{2}(c) \sigma(c) c+\sigma^{2}(d) c+\sigma^{2}(c) \sigma(d)-a_{3}(\sigma(d)+\sigma(c) c)-a_{2} c-a_{1}=0,
$$

and

$$
\sigma^{2}(d) d+\sigma^{2}(c) \sigma(c) d-a_{3} \sigma(c) d-a_{2} d-a_{0}=0 .
$$

Propositions 22 and 29 together with (11) yield:

Theorem 30. $f(t)=t^{4}-a_{3} t^{3}-a_{2} t^{2}-a_{1} t-a_{0} \in R=D[t ; \sigma]$ is irreducible if and only if

$$
\sigma^{3}(b) \sigma^{2}(b) \sigma(b) b+a_{3} \sigma^{2}(b) \sigma(b) b+a_{2} \sigma(b) b+a_{1} b+a_{0} \neq 0,
$$

and

$$
\begin{aligned}
& \sigma^{3}(b) \sigma^{2}(b) \sigma(b) b+\sigma^{3}(b) \sigma^{2}(b) \sigma(b) a_{3}+ \\
& \sigma^{3}(b) \sigma^{2}(b) \sigma\left(a_{2}\right)+\sigma^{3}(b) \sigma^{2}\left(a_{1}\right)+\sigma^{3}\left(a_{0}\right) \neq 0,
\end{aligned}
$$

for all $b \in D$, and for every $c, d \in D$, we have

$$
\sigma^{2}(c) \sigma(c) c+\sigma^{2}(d) c+\sigma^{2}(c) \sigma(d)+a_{3}(\sigma(d)+\sigma(c) c)+a_{2} c+a_{1} \neq 0,
$$

or

$$
\sigma^{2}(d) d+\sigma^{2}(c) \sigma(c) d+a_{3} \sigma(c) d+a_{2} d+a_{0} \neq 0 .
$$

I.e., $f(t)$ is irreducible if and only if (16) and (17) and ((18) or (19)) hold.

Proof. $f(t)$ is irreducible if and only if $(t-b) \nmid_{r} f(t)$ for all $b \in D,(t-b) \nmid_{l} f(t)$ for all $b \in D$ and $\left(t^{2}-c t-d\right) \nmid_{r} f(t)$ for all $c, d \in D$. Therefore the result follows from (11), Propositions 22 and 29 .

Lemma 31. Let $f(t)=t^{4}-a \in R$. Suppose $\left.(t-b)\right|_{r} f(t)$, then $f(t)=\left(t+\sigma^{3}(b)\right)\left(t^{2}+\right.$ $\left.\sigma^{2}(b) \sigma(b)\right)(t-b)$ and $f(t)=\left(t^{2}+\sigma^{3}(b) \sigma^{2}(b)\right)(t+\sigma(b))(t-b)$ are factorisations of $f(t)$. In particular, $(t+\sigma(b))(t-b)=t^{2}-\sigma(b) b$ also right divides $f(t)$.

Proof. Multiplying out these factorisations gives $t^{4}-\sigma^{3}(b) \sigma^{2}(b) \sigma(b) b$ which is equal to $f(t)$ by $(11)$.

Hence if $f(t)=t^{4}-a$ has a right linear divisor then it also has a right quadratic divisor and Theorem 30 simplifies to:

Corollary 32. $f(t)=t^{4}-a \in R$ is reducible if and only if

$$
\sigma^{2}(c) \sigma(c) c+\sigma^{2}(d) c+\sigma^{2}(c) \sigma(d)=0 \quad \text { and } \quad \sigma^{2}(d) d+\sigma^{2}(c) \sigma(c) d=a,
$$

for some $c, d \in D$.

Proof. By Corollary 23, $f(t)$ has a right linear divisor if and only if it has a left linear divisor. Moreover if $f(t)$ has a right linear divisor then it also has a quadratic right divisor by Lemma 31, therefore $f(t)$ is reducible if and only if $\left.\left(t^{2}-c t-d\right)\right|_{r} f(t)$ for some $c, d \in D$. The result now follows from Proposition 29. 
5.3. Examples in $\mathbb{F}_{p^{h}}[t ; \sigma]$. Let $K=\mathbb{F}_{p^{h}}$ be a finite field of order $p^{h}$ for some prime $p$ and $\sigma$ be a non-trivial $\mathbb{F}_{p^{-}}$-automorphism of $K$. This means $\sigma: K \rightarrow K, k \mapsto k^{p^{r}}$, for some $r \in\{1, \ldots, h-1\}$. Here $\sigma$ has order $n=h / \operatorname{gcd}(r, h)$ and $\operatorname{Gal}(K / \operatorname{Fix}(\sigma))=\langle\sigma\rangle$. Algorithms for efficiently factorising polynomials in $\mathbb{F}_{p^{h}}[t ; \sigma]$ exist, see [13] or more recently [10].

Lemma 33. $\operatorname{gcd}\left(p^{h}-1, p^{r}-1\right)=p^{\operatorname{gcd}(h, r)}-1$.

Proof. Let $d=\operatorname{gcd}(r, h)$ so that $h=d n$. We have

$$
p^{h}-1=\left(p^{d}-1\right)\left(p^{d(n-1)}+\ldots+p^{d}+1\right),
$$

therefore $p^{h}-1$ is divisible by $p^{d}-1$. A similar argument shows $\left(p^{d}-1\right) \mid\left(p^{r}-1\right)$. Suppose that $c$ is a common divisor of $p^{h}-1$ and $p^{r}-1$, this means $p^{h} \equiv p^{r} \equiv 1 \bmod (c)$. Write $d=h x+r y$ for some integers $x, y$, then we have

$$
p^{d}=p^{h x+r y}=\left(p^{h}\right)^{x}\left(p^{r}\right)^{y} \equiv 1 \bmod (c)
$$

which implies $c \mid\left(p^{d}-1\right)$ and hence $p^{d}-1=\operatorname{gcd}\left(p^{h}-1, p^{r}-1\right)$.

Given $k \in K^{\times}$, we have $k \in \operatorname{Fix}(\sigma)$ if and only if $k^{p^{r}-1}=1$, if and only if $k$ is a $\left(p^{r}-1\right)^{\text {th }}$ root of unity. There are $\operatorname{gcd}\left(p^{r}-1, p^{h}-1\right)$ such roots of unity in $K$, thus

$$
|\operatorname{Fix}(\sigma)|=\operatorname{gcd}\left(p^{r}-1, p^{h}-1\right)+1=p^{\operatorname{gcd}(r, h)}
$$

by Lemma 33 and so $\operatorname{Fix}(\sigma) \cong \mathbb{F}_{q}$ where $q=p^{\operatorname{gcd}(r, h)}$.

Proposition 34. (i) Suppose $n \in\{2,3\}$, then $f(t)=t^{n}-a \in K[t ; \sigma]$ is irreducible if and only if $a \in K \backslash \operatorname{Fix}(\sigma)$.

(ii) Suppose $n$ is a prime and $n \mid(q-1)$. Then $f(t)=t^{n}-a \in K[t ; \sigma]$ is irreducible if and only if $a \in K \backslash \operatorname{Fix}(\sigma)$.

In particular, in both (i) and (ii), there are precisely $p^{h}-q$ irreducible polynomials in $K[t ; \sigma]$ of the form $t^{n}-a$ for some $a \in K$.

Proof. (i) $f(t)$ is irreducible if and only if $\prod_{l=0}^{n-1} \sigma^{l}(b)=N_{K / \operatorname{Fix}(\sigma)}(b) \neq a$ for all $b \in K$ by Theorem 24 or Corollary 25, where $N_{K / \operatorname{Fix}(\sigma)}$ is the field norm. It is well-known that as $K$ is a finite field, $N_{K / \operatorname{Fix}(\sigma)}: K^{\times} \rightarrow \operatorname{Fix}(\sigma)^{\times}$is surjective and so $f(t)$ is irreducible if and only if $a \notin \operatorname{Fix}(\sigma)$. There are $p^{h}-q$ elements in $K \backslash \operatorname{Fix}(\sigma)$, hence there are precisely $p^{h}-q$ irreducible polynomials of the form $t^{n}-a$ for some $a \in K$.

(ii) $\operatorname{Fix}(\sigma) \cong \mathbb{F}_{q}$ contains a primitive $n^{\text {th }}$ root of unity because $n \mid(q-1)$ [23, Proposition II.2.1]. The rest of the proof is similar to (i) but uses Theorem 28.

Let $a, b \in K$ and recall $\left.(t-b)\right|_{r}\left(t^{m}-a\right)$ is equivalent to $a=\sigma^{m-1}(b) \cdots \sigma(b) b=b^{s}$ by (11) where $s=\sum_{j=0}^{m-1} p^{r j}=\left(p^{m r}-1\right) /\left(p^{r}-1\right)$. Suppose $z$ generates the multiplicative group $K^{\times}$. Writing $b=z^{l}$ for some $l \in \mathbb{Z}$ yields $\left.(t-b)\right|_{r}\left(t^{m}-a\right)$ if and only if $a=z^{l s}$. This implies the following:

Proposition 35. Let $f(t)=t^{m}-a \in K[t ; \sigma]$ and write $a \in K$ as $a=z^{u}$ for some $u \in\left\{0, \ldots, p^{h}-2\right\}$.

(i) $(t-b) \nmid_{r} f(t)$ for all $b \in K$ if and only if $u \notin \mathbb{Z} s \bmod \left(p^{h}-1\right)$.

(ii) If $m \in\{2,3\}$ then $f(t)$ is irreducible if and only if $u \notin \mathbb{Z} s \bmod \left(p^{h}-1\right)$. 
(iii) Suppose $m$ is a prime divisor of $(q-1)$, then $f(t)$ is irreducible if and only if $u \notin$ $\mathbb{Z} s \bmod \left(p^{h}-1\right)$.

Proof. (i) $(t-b) \nmid_{r} f(t)$ for all $b \in K$ if and only if $a=z^{u} \neq z^{l s}$ for all $l \in \mathbb{Z}$, if and only if $u \notin \mathbb{Z} s \bmod \left(p^{h}-1\right)$.

(ii) $f(t)$ has a left linear divisor if and only if it has a right linear divisor by Corollary 23. Therefore if $m \in\{2,3\}$ then $f(t)$ is irreducible if and only if $(t-b) \nmid_{r} f(t)$ for all $b \in K$ and so the assertion follows by (i).

(iii) If $m$ is a prime divisor of $(q-1)$ then $\operatorname{Fix}(\sigma) \cong \mathbb{F}_{q}$ contains a primitive $m^{\text {th }}$ root of unity. Therefore the result follows by (i) and Theorem 28 .

Corollary 36. (i) There exists $a \in K$ such that $(t-b) \nmid_{r}\left(t^{m}-a\right)$ for all $b \in K$ if and only if $\operatorname{gcd}\left(s, p^{h}-1\right)>1$.

(ii) $[30,(22)]$ Suppose $m \in\{2,3\}$ or $m$ is a prime divisor of $(q-1)$. Then there exists $a \in K^{\times}$such that $t^{m}-a \in K[t ; \sigma]$ is irreducible if and only if $\operatorname{gcd}\left(s, p^{h}-1\right)>1$.

Proof. There exists $u \in\left\{0, \ldots, p^{h}-2\right\}$ such that $u \notin \mathbb{Z} s \bmod \left(p^{h}-1\right)$, if and only if $s$ does not generate $\mathbb{Z}_{p^{h}-1}$, if and only if $\operatorname{gcd}\left(s, p^{h}-1\right)>1$. Hence the result follows by Proposition 35 .

Corollary 37. Suppose $p \equiv 1 \bmod m$.

(i) There exists $a \in K$ such that $(t-b) \nmid_{r}\left(t^{m}-a\right)$ for all $b \in K$.

(ii) If $p$ is an odd prime, then there exists $a \in K^{\times}$such that $t^{2}-a \in K[t ; \sigma]$ is irreducible.

(iii) If $m=3$, then there exists $a \in K^{\times}$such that $t^{3}-a \in K[t ; \sigma]$ is irreducible.

(iv) Suppose $m$ is a prime divisor of $(q-1)$, then there exists $a \in K^{\times}$such that $t^{m}-a \in$ $K[t ; \sigma]$ is irreducible.

Proof. We have

$$
s \bmod m=\sum_{i=0}^{m-1}\left(p^{r i} \bmod m\right) \bmod m=\left(\sum_{i=0}^{m-1} 1\right) \bmod m=0,
$$

and $p^{h} \equiv 1 \bmod m$. This means $m \mid s$ and $m \mid\left(p^{h}-1\right)$, therefore $\operatorname{gcd}\left(s, p^{h}-1\right) \geq m$ and so the assertion follows by Corollary 36 .

\section{IrREducibility CRITERIA FOR POLYNOMIALS OF DEGREe TWO AND THREE AND FOR}

$$
t^{m}-a \text { IN } D[t ; \sigma, \delta]
$$

In this Section we generalize some results from Section 5 to polynomials in $R=D[t ; \sigma, \delta]$, where $D$ is a division ring with center $F$ and $\sigma$ an endomorphism of $D$. We recursively define a sequence of maps $N_{i}: D \rightarrow D, i \geq 0$, by

$$
N_{0}(b)=1, N_{i+1}(b)=\sigma\left(N_{i}(b)\right) b+\delta\left(N_{i}(b)\right),
$$

i.e., $N_{1}(b)=b, N_{2}(b)=\sigma(b) b+\delta(b), \ldots$

Let $f(t)=t^{m}-\sum_{i=0}^{m-1} a_{i} t^{i} \in R$. Then $\left.(t-b)\right|_{r} f(t)$ is equivalent to

$$
N_{m}(b)-\sum_{i=0}^{m-1} a_{i} N_{i}(b)=0
$$


[25, Lemma 2.4].

If $\sigma$ is an automorphism of $D$, we can also view $R=D[t ; \sigma, \delta]$ as a right polynomial ring. In particular, this means we can write $f(t)=t^{m}-\sum_{i=0}^{m-1} a_{i} t^{i} \in R$ in the form $f(t)=t^{m}-\sum_{i=0}^{m-1} t^{i} a_{i}^{\prime}$ for some uniquely determined $a_{i}^{\prime} \in D$. To find the remainder after left division of $f(t)$ by $(t-b)$, we recursively define a sequence of maps $M_{i}: D \rightarrow D, i \geq 0$, by

$$
M_{i+1}(b)=b \sigma^{-1}\left(M_{i}(b)\right)-\delta\left(\sigma^{-1}\left(M_{i}(b)\right)\right), M_{0}(b)=1,
$$

that is $M_{0}(b)=1, M_{1}(b)=b, M_{2}(b)=b \sigma^{-1}(b)-\delta\left(\sigma^{-1}(b)\right), \ldots$

Proposition 38. Suppose $\sigma$ is an automorphism of $D$. Then $\left.(t-b)\right|_{l} f(t)$ is equivalent to $M_{m}(b)-\sum_{i=0}^{m-1} M_{i}(b) a_{i}^{\prime}=0$. In particular, $\left.(t-b)\right|_{l}\left(t^{m}-a\right)$ if and only if $M_{m}(b) \neq a$.

Proof. We first show $t^{n}-M_{n}(b) \in(t-b) R$ for all $b \in D$ and $n \geq 0$ : If $n=0$ then $t^{0}-M_{0}(b)=1-1=0 \in(t-b) R$ as required. Suppose inductively $t^{n}-M_{n}(b) \in(t-b) R$ for some $n \geq 0$, then

$$
\begin{aligned}
& t^{n+1}-M_{n+1}(b)=t^{n+1}-b \sigma^{-1}\left(M_{n}(b)\right)+\delta\left(\sigma^{-1}\left(M_{n}(b)\right)\right) \\
& =t^{n+1}+(t-b) \sigma^{-1}\left(M_{n}(b)\right)-t \sigma^{-1}\left(M_{n}(b)\right)+\delta\left(\sigma^{-1}\left(M_{n}(b)\right)\right) \\
& =t^{n+1}+(t-b) \sigma^{-1}\left(M_{n}(b)\right)-M_{n}(b) t-\delta\left(\sigma^{-1}\left(M_{n}(b)\right)\right)+\delta\left(\sigma^{-1}\left(M_{n}(b)\right)\right) \\
& =(t-b) \sigma^{-1}\left(M_{n}(b)\right)+\left(t^{n}-M_{n}(b)\right) t \in(t-b) R,
\end{aligned}
$$

as $t^{n}-M_{n}(b) \in(t-b) R$. Therefore $t^{n}-M_{n}(b) \in(t-b) R$ for all $b \in D, n \geq 0$ by induction.

As a result, there exists $q_{i}(t) \in R$ such that $t^{i}=(t-b) q_{i}(t)+M_{i}(b)$, for all $i \in\{0, \ldots, m\}$. Multiplying on the right by $a_{i}^{\prime}$ and summing over $i$ yields

$$
f(t)=(t-b) q(t)+M_{m}(b)-\sum_{i=0}^{m-1} M_{i}(b) a_{i}^{\prime},
$$

for some $q(t) \in R$.

Theorem 39. (i) $f(t)=t^{2}-a_{1} t-a_{0} \in D[t ; \sigma, \delta]$ is irreducible if and only if $\sigma(b) b+\delta(b)-$ $a_{1} b-a_{0} \neq 0$ for all $b \in D$.

(ii) Suppose $\sigma$ is an automorphism of $D$ and $f(t)=t^{3}-a_{2} t^{2}-a_{1} t-a_{0} \in D[t ; \sigma, \delta]$. Write $f(t)=t^{3}-t^{2} a_{2}^{\prime}-t a_{1}^{\prime}-a_{0}^{\prime}$ for some unique $a_{0}^{\prime}, a_{1}^{\prime}, a_{2}^{\prime} \in D$. Then $f(t)$ is irreducible if and only if

$$
N_{3}(b)-\sum_{i=0}^{2} a_{i} N_{i}(b) \neq 0 \text { and } M_{3}(b)-\sum_{i=0}^{2} M_{i}(b) a_{i}^{\prime} \neq 0
$$

for all $b \in D$.

Proof. (i) $f(t)$ is irreducible if and only if it has no right linear factors, if and only if

$$
N_{2}(b)-a_{1} N_{1}(b)-a_{0} N_{0}(b)=\sigma(b) b+\delta(b)-a_{1} b-a_{0} \neq 0,
$$

for all $b \in D$ by (20).

(ii) $f(t)$ is irreducible if and only if it has no left or right linear factors, if and only if (21) holds for all $b \in D$ by (20) and Proposition 38 . 
We can thus generalize Theorem 28:

Theorem 40. Suppose $m$ is prime, $\operatorname{char}(D) \neq m$ and $F \cap \operatorname{Fix}(\sigma)$ contains a primitive mth root of unity $\omega$. Then $f(t)=t^{m}-a \in D[t ; \sigma, \delta]$ is irreducible if and only if $N_{m}(b) \neq a$ for all $b \in D$.

Proof. Recall that $\delta\left(b^{n}\right)=\sum_{i=0}^{n-1} \sigma(b)^{i} \delta(b) b^{n-1-i}$ for all $b \in D, n \geq 1$ by [16, Lemma 1.1] and so

$$
\begin{aligned}
0 & =\delta(1)=\delta\left(\omega^{m}\right)=\sum_{i=0}^{m-1} \sigma(\omega)^{i} \delta(\omega) \omega^{m-1-i}=\sum_{i=0}^{m-1} \omega^{i} \delta(\omega) \omega^{m-1-i} \\
& =\sum_{i=0}^{m-1} \delta(\omega) \omega^{m-1}=\delta(\omega) \omega^{m-1} m,
\end{aligned}
$$

where we have used $\omega \in F \cap \operatorname{Fix}(\sigma)$. Therefore $\omega \in \operatorname{Const}(\delta)$ because $\operatorname{char}(D) \neq m$, hence also $\omega^{i} \in \operatorname{Const}(\delta)$ and so $(\omega t)^{i}=\omega^{i} t^{i}$ for all $i \in\{1, \ldots, m\}$. Furthermore if $b \in D$, then $(t-b) \nmid_{r} f(t)$ is equivalent to $N_{m}(b) \neq a$ by $(20)$. The proof now follows exactly as in Theorem 24.

Corollary 41. Suppose $\operatorname{char}(D) \neq 3, \sigma=i d$ and $F \cap \operatorname{Fix}(\sigma)$ contains a primitive third root of unity. Then $f(t)=t^{3}-a \in D[t ; \delta]$ is irreducible if and only if

$$
N_{3}(b)=b^{3}+2 \delta(b) b+b \delta(b)+\delta^{2}(b) \neq a,
$$

for all $b \in D$.

\section{REFERENCES}

[1] A. S. Amitsur, Non-commutative cyclic fields. Duke Math. J. 21 (1954), 87-105.

[2] J. Bergen, M. Giesbrecht, P. N. Shivakumar, Y. Zhang, Factorizations for difference operators. Advances in Difference Equations 57 (2015), DOI 10.1186/s13662-015-0402-1 (Springer)

[3] N. Boubaki, "Éléments de mathématique. Fasc. XXIII. Algèbre; Chapitre 8, modules e anneaux semisimples." Hermann, 1973.

[4] C. Brown, "Petit algebras and their automorphisms." PhD Thesis, University of Nottingham, 2018. Online at arXiv:1806.00822 [math.RA]

[5] C. Brown, S. Pumplün, The automorphisms of Petit's algebras. Comm. Algebra 46 (2) (2018), 834-849.

[6] C. Brown, S. Pumplün, Nonassociative cyclic extensions of fields and central simple algebras. J. Pure Applied Algebra 223 (6) 2019, 2401-2412.

[7] R. H. Bruck, Contributions to the theory of loops. Trans. AMS 60 (2) (1946), 245-345.

[8] J. Carcanague, Ideaux bilaterales d'un anneau de polynomes non commutatifs sur un corps. J. Algebra (18) $1971,1-18$.

[9] J. Carcanague, Quelques resultats sur les anneaux de Ore. C. R. Acad. Sci. Paris Sr. A-B 269 (1969), A749-A752.

[10] X. Caruso, J. Le Borgne, A new faster algorithm for factoring skew polynomials over finite fields. J. Symb. Comp. 79 (2017), 411-443.

[11] R. C. Churchill, Y. Zhang, Irreducibility criteria for skew polynomials. J. Algebra 322 (2009), 3797-3822.

[12] P. M. Cohn, "Skew fields. Theory of general division rings." Encyclopedia of Mathematics and its Applications. Cambridge University Press, 1995.

[13] M. Giesbrecht, Factoring in skew-polynomial rings over finite fields. J. Symbolic Comput. 26 (4) (1998), 463-486. 
[14] M. Giesbrecht, Y. Zhang, Factoring and decomposing Ore polynomials over $\mathbb{F}_{q}(t)$. Proceedings of the 2003 International Symposium on Symbolic and Algebraic Computation, 127134, ACM, New York, 2003.

[15] J. Gòmez-Torrecillas, Basic module theory over non-commutative rings with computational aspects of operator algebras. With an appendix by V. Levandovskyy. Lecture Notes in Comput. Sci. 8372, Algebraic and algorithmic aspects of differential and integral operators, Springer, Heidelberg (2014) 23-82.

[16] K. Goodearl, Prime ideals in skew polynomial rings and quantized Weyl algebras. J. Algebra 150 (2) (1992), 324-377.

[17] J. Gòmez-Torrecillas, F. J. Lobillo, G. Navarro, Computing the bound of an Ore polynomial. Applications to factorization. J. Symbolic Comp. 2018, online at https://doi.org/10.1016/j.jsc.2018.04.018

[18] A. Granja, C. Martinez, C. Rodriguez, Real Valuations on Skew Polynomial Rings. Algebras and Representation Theory 17 (5) (2014), 1413-1436.

[19] Hungerford, "Algebra." Vol. 73 of Graduate Texts in Mathematics. Springer Verlag, New York, 1980.

[20] N. Jacobson, "Finite-dimensional division algebras over fields." Springer Verlag, Berlin-Heidelberg-New York, 1996.

[21] N. Jacobson, Pseudo-linear transformations. Ann. of Math. (2) 38 (1937), no. 2, 484-507.

[22] N. Jacobson, "The Theory of Rings", AMS, Providence, RI, 1943

[23] N. Koblitz, "A course in number theory and cryptography." Vol. 114, Springer Science and Business Media, 1994.

[24] M. Lavrauw, J. Sheekey, Semifields from skew-polynomial rings. Adv. Geom. 13 (4) (2013), 583-604.

[25] T. Y. Lam, A. Leroy, Vandermonde and Wroskian matrices over division rings. J. Alg. 19(2)(1988), 308-336.

[26] T. Y. Lam, A. Leroy, Algebraic conjugacy classes and skew-polynomial rings. In Perspectives in Ring Theory, 153-203, Springer, 1988.

[27] T. Y. Lam, K. H. Leung, A. Leroy, J. Matczuk, Invariant and semi-invariant polynomials in skew polynomial rings. Ring theory 1989 (Ramat Gan and Jerusalem, 1988/1989), 247-261, Israel Math. Conf. Proc., 1, Weizmann, Jerusalem, 1989.

[28] B. Lemonnier, Dimension de Krull et codeviations, quelques applications en theorie des modules. PhD Thesis, Poitiers, 1984.

[29] O. Ore, Theory of noncommutative polynomials. Annals of Math. 34 (3) (1933), 480-508.

[30] J.-C. Petit, Sur certains quasi-corps généralisant un type d'anneau-quotient. Séminaire Dubriel. Algèbre et théorie des nombres 20 (1966 - 67), 1-18.

[31] J.-C. Petit, Sur les quasi-corps distributifes à base momogène. C. R. Acad. Sc. Paris 266 (1968), Série A, 402-404.

[32] S. Pumplün, Factoring skew polynomials over Hamilton's quaternion algebra and the complex numbers. J. Algebra 427 (2015), 20-29.

[33] S. Pumplün, Corrigendum to: "Factoring skew polynomials over Hamilton's quaternion algebra and the complex numbers" [J. Algebra 427 (2015), 20-29]. Online at http://agt2.cie.uma.es/ loos/jordan/

[34] S. Pumplün, Nonassociative differential extensions of characteristic $p$. Results in Mathematics 72 (1-2) (2017), 245-262.

[35] S. Pumplün, Finite nonassociative algebras obtained from skew polynomials and possible applications to $(f, \sigma, \delta)$-codes. Adv. Math. Comm. 11 (3) (2017), 615-634. doi:10.3934/amc.2017046

[36] S. Pumplün, Tensor products of nonassociative cyclic algebras. Journal of Algebra 451 (2016), 145-165.

[37] S. Pumplün, Algebras whose right nucleus is a central simple algebra. J. Pure and Applied Algebra 222 (9) (2018), 2773-2783. https://doi.org/10.1016/j.jpaa.2017.10.019

[38] S. Pumplün, How to obtain lattices from $(f, \sigma, \delta)$-codes via a generalization of Construction A. Appl. Algebra Engrg. Comm. Comput. 29 (4) (2018), 313-333.

[39] S. Pumplün, Quotients of orders in algebras obtained from skew polynomials with applications to coding theory. Comm. Algebra 46 (11) (2018), 5053-5072.

[40] L. Rónyai, Factoring polynomials over finite fields. J. Algorithms 9 (3) (1988), 391-400.

[41] R. D. Schafer, "An Introduction to Nonassociative Algebras." Dover Publ., Inc., New York, 1995. 
Email address: christian_jb@hotmail.co.uk; susanne.pumpluen@nottingham.ac.uk

School of Mathematical Sciences, University of Nottingham, University Park, Nottingham NG7 2RD, United Kingdom 